\title{
Bioanalysis
}

\section{Chemical derivatization-based LC-MS for metabolomics: advantages and challenges}

\author{
"Analyzing as many metabolites as possible in a single LC-MS run is \\ challenging..."
}

First draft submitted: 15 July 2016; Accepted for publication: 25 July 2016; Published online: 15 August 2016

Keywords: chemical derivatization $\bullet$ LC-MS • metabolomics

\section{General aspects}

Metabolomics is a systemic methodology aiming at measuring all altered endogenous low-molecular-weight metabolites (usually $<1500 \mathrm{Da}$ ) in cell, tissue or the whole organism. Generally, according to the number of detected metabolites as well as the reliability of quantification method used, metabolomics can be categorized into two strategies: untargeted and targeted. The broad scale of measured metabolites is the goal of untargeted metabolomics, while as for targeted metabolomics, much more attentions will be given to the quantitative analysis of a subset of metabolites in a selected metabolic pathway. Each strategy has their own advantages and limitations. Untargeted metabolomics was usually performed first to screen out the key differential metabolites and the related metabolic pathway. A followed-up targeted metabolomics could be further conducted to validate these findings and uncover more detailed relationship among differential metabolites.

MS and NMR are two main analytical technologies applied in metabolomics research. MS is surpassing the use of NMR at a 5:2 ratio and is becoming the dominant analytical platform due to its inherent high sensitivity, rapid improvement of instruments and flexible compatibility with chromatographic separation [1]. LC and GC are both effective separation strategies for metabolomics when combined with MS. Because of the differences in separation and ionization mechanisms, both platforms hold their own strengths and limitations. GC-MS is always applied to analyze volatile compounds, derivatization is an indispensable part to transform analytes into volatile and thermally stable derivatives. However, multipeak and multi-origination phenomena derived from derivatization-based GC-MS could influence the interpretation of metabolic pathways [2]. LC-MS has become a preferred analytical platform for its various separation mechanisms and 'soft' ionization. Especially the introduction of UPLC, have profound implications for high-throughput metabolomics analysis.

The metabolome is the terminal product of genome and proteome, consisting of thousands of metabolites with a wide concentration range and various physicochemical properties in complex biological samples. Analyzing as many metabolites as possible in a single LC-MS run is challenging as a large number of compounds are poorly ionizable or have poor retention time. In this regard, chemical derivatization could introduce novel functional groups and transform analytes into MS-detectable and LC-separable derivatives. Large quantity of emerging derivatization reagents for metabolites have been synthesized over the last decade [3], they were designed for respective metabolites with different functional groups such as amines, phenols, carboxylic acids, steroids, thiols and alcohols [4]. The enhancement of detectability is the main purpose of derivatization-based

\section{Ruiqi Jiang}

Key Laboratory of Drug Quality Control \& Pharmacovigilance, China Pharmaceutical University, Nanjing 210009, PR China and

State Key Laboratory of Natural Medicine, China Pharmaceutical University, Nanjing 210009, P. R. China

\section{Yu Jiao}

State Key Laboratory of Natural Medicine, China Pharmaceutical University, Nanjing 210009, PR China and

Department of Organic Chemistry, China Pharmaceutical University, Nanjing 210009, PR China

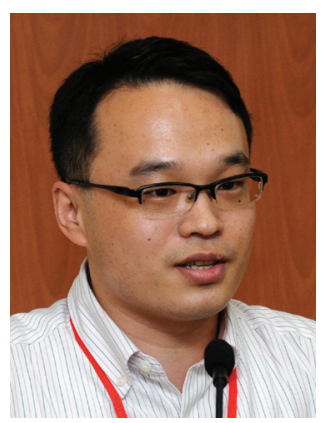

\section{Fengguo $\mathrm{Xu}$}

Author for correspondence: Key Laboratory of Drug Quality Control \& Pharmacovigilance, China Pharmaceutical University, Nanjing 210009, PR China and

State Key Laboratory of Natural Medicine, China Pharmaceutical University, Nanjing 210009, PR China fengguoxu@gmail.com

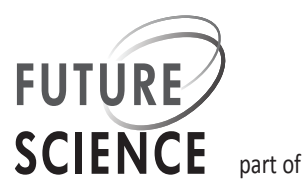


LC-MS through increasing evaporation and nebulization efficiency, introducing chargeable or easily ionizable moieties and aiding fragmentation in the MRM mode [4-6]. Interestingly, a new strategy that integrates several subsets of metabolites with different functional groups together by derivatization-based LC-MS for untargeted metabolomics has been proposed [7]. In this way, the coverage of metabolites, the selectivity and sensitivity could be improved significantly compared with conventional LC-MS-based metabolomics.

\section{The design \& selection of optimal derivatization reagents}

With the increasing demand of analyzing thousands of metabolites in a single LC-MS run, various derivatization reagents with diverse chemical structures and properties were designed [8]. However, the lack of systematic study in this field limits its wide application. So far there is still no mature theory to guide the design and selection of new reagents. To obtain optimal derivatization reagents, several points should be taken into consideration:

- The activity of reacting groups;

- The stability of derivatives;

- The efficiency of ionization;

- The hydrophobicity of derivatives.

Physicochemical parameters, such as $\mathrm{pK}_{\mathrm{a}}, \log \mathrm{P}$, polar surface area [9], proton affinity [10], WAPS [11], may reflect the above properties to some extent so as to aid to design and select the derivatization reagents for LC-MS. Prior to analyzing the actual samples, these parameters could help us to evaluate the efficiency of the candidate reagents.

\section{"So far there is still no mature theory to guide the design and selection of new reagents."}

As the process of ionization in MS is very complex, the above-mentioned parameter alone cannot be used to assess and predict the performance of derivatization reagents. How to integrate the above physicochemical parameters reasonably to guide the design of chemical derivatization reagents and selection is still an issue that needs to be addressed.

\section{Challenges in metabolite identification}

Metabolite identification is still at a bottleneck in terms of LC-MS-based metabolomics research. It is fairly difficult to convert enormous analytical data into meaningful biological information. Sumner et al. divided metabolite identification into four levels: 'positively identified compounds, putatively identified compounds, compounds putatively identified in a compound class and unknown compounds' [12]. It is estimated that approximately 5000 compounds with commercially available authentic standards can be positively identified among 200,000 metabolites at the percentage of $2.5 \%$ [13]. The remaining $97.5 \%$ metabolites could only be putatively identified referring to the databases or the structure analogs that were commercially available. In recent years, the databases for LC-MS metabolomics including HMDB [14], METLIN [15], MassBank [16] have been accessed freely, which enhance the feasibility for metabolite identification. As for derivatization-based LC-MS, the introduction of a known-shared chemical structure into the original compounds could also add values to the identification of metabolites. Li and colleagues reported a locally constructed library based on dansylation LC-MSbased metabolomics that covers amine- and phenolcontaining metabolites [17], extending the strength of chemical derivatization on metabolite identification.

As described above, there are about $97.5 \%$ metabolites that cannot be positively identified using commercially available authentic standards. How to predict the possible structure of them is a challenge in metabolite identification. In LC-MS, diagnostic ions derived from a set of known patterns are usually used to predict the structure of unknown metabolites, especially for compounds that are structural analogs. Chemical derivatization may be an alternative way to facilitate the prediction of unknown key metabolites. Generally, the specific fragment of derivatized compound induced by CID originates from derivatization reagent moiety, and the residual moiety is related to intact analyte. Thus, based on the fragmentation patterns of the known residual moieties, the possible chemical structures of unknown metabolites could be recognized without authentic standards.

\section{Conclusion \& future perspective}

Chemical derivatization-based LC-MS is an emerging strategy for metabolomics research. The introduction of chemical moiety could extend the metabolite coverage, improve the LC separation and MS detection for both untargeted and targeted metabolomics. However, there are still many challenges existing in this new strategy. General principles for the design and selection of optimal derivatization reagents should be summarized to guide the researchers. In order to promote the identification of unknown metabolites, shared databases for derivatized compounds should be developed, although this may be a tough project. 
Although effective, the limitations of chemical derivatization such as time-taken, introduction of variations and by-product formation must be considered and evaluated. In the near future, with the development of new specific derivative reagents that have high reaction selectivity for LC-MS, the application of metabolomics in, for example, the fields of pharmaceutical research, food-safety evaluation, environmental monitoring and precision medicine study will be greatly extended.

\section{References}

1 Cajka T, Fiehn O. Toward merging untargeted and targeted methods in mass spectrometry-based metabolomics and lipidomics. Anal. Chem. 88(1), 524-545 (2015).

2 Xu F, Zou L, Ong CN. Experiment-originated variations, and multi-peak and multi-origination phenomena in derivatization-based GC-MS metabolomics. TrAC Trends Anal. Chem. 29(3), 269-280 (2010).

3 Higashi T, Ogawa S. Isotope-coded ESI-enhancing derivatization reagents for differential analysis, quantification and profiling of metabolites in biological samples by LC/MS: a review. J. Pharm. Biomed. Anal. doi: 10.1016/j. jpba.2016.04.033 (2016) (Epub ahead of print).

4 Xu F, Zou L, Liu Y, Zhang Z, Ong CN. Enhancement of the capabilities of liquid chromatography-mass spectrometry with derivatization: general principles and applications. Mass Spectrom. Rev. 30(6), 1143-1172 (2011).

5 He Y, Zhao X-E, Zhu S et al. In situ derivatizationultrasound-assisted dispersive liquid-liquid microextraction for the determination of neurotransmitters in Parkinson's rat brain microdialysates by ultra high performance liquid chromatography-tandem mass spectrometry. J. Chromatogr. A 1458, 70-81 (2016).

6 Huang Q, Liu D, Xin B et al. Quantification of monosialogangliosides in human plasma through chemical derivatization for signal enhancement in LC-ESI-MS. Anal. Chim. Acta 929, 31-38 (2016).

7 Luo X, Zhao S, Huan T et al. High-performance chemical isotope labeling liquid chromatography-mass spectrometry for profiling the metabolomic reprogramming elicited by ammonium limitation in yeast. J. Proteome Res. 15(5), 1602-1612 (2016).

\section{Financial \& competing interests disclosure}

The authors have no relevant affiliations or financial involvement with any organization or entity with a financial interest in or financial conflict with the subject matter or materials discussed in the manuscript. This includes employment, consultancies, honoraria, stock ownership or options, expert testimony, grants or patents received or pending, or royalties.

No writing assistance was utilized in the production of this manuscript.

8 Zhu Y, Deng P, Zhong D. Derivatization methods for LC-MS analysis of endogenous compounds. Bioanalysis 7(19), 2557-2581 (2015).

9 Niwa M. Chemical derivatization as a tool for optimizing MS response in sensitive LC-MS/MS bioanalysis and its role in pharmacokinetic studies. Bioanalysis 4(2), 213-220 (2012).

10 Hunter EP, Lias SG. Evaluated gas phase basicities and proton affinities of molecules: an update. J. Phys. Chem. Ref. Data 27(3), 413-656 (1998).

11 Kruve A, Kaupmees K, Liigand J, Leito I. Negative electrospray ionization via deprotonation: predicting the ionization efficiency. Anal. Chem. 86(10), 4822-4830 (2014).

12 Sumner LW, Amberg A, Barrett D et al. Proposed minimum reporting standards for chemical analysis. Metabolomics 3(3), 211-221 (2007).

13 Wishart DS. Advances in metabolite identification. Bioanalysis 3(15), 1769-1782 (2011).

14 Wishart DS, Jewison T, Guo AC et al. HMDB 3.0 - the human metabolome database in 2013. Nucl. Acids Res. 41, D801-D807 (2012).

15 Smith CA, O'maille G, Want EJ et al. METLIN: a metabolite mass spectral database. Ther. Drug Monit. 27(6), 747-751 (2005).

16 Horai H, Arita M, Kanaya S et al. MassBank: a public repository for sharing mass spectral data for life sciences. J. Mass Spectrom. 45(7), 703-714 (2010).

17 Huan T, Tang C, Li R, Shi Y, Lin G, Li L. MyCompoundID MS/MS search: metabolite identification using a library of predicted fragment-ion-spectra of 383,830 possible human metabolites. Anal. Chem. 87(20), 10619-10626 (2015). 